\title{
Bilan de santé actuel et rétrospectif du sapin (Abies alba Mill.) dans les Vosges. Etude écologique et dendrochronologique
}

\author{
M. BECKER \\ avec la collaboration technique de F. GEREMIA et R. SCHIPFER \\ INRA, Laboratoire de Phyto-écologie forestière \\ Centre de Recherches de Nancy, BP 35, Champenoux, F 54280 Seichamps
}

\section{Résumé}

Le dispositif retenu comprend 196 placettes réparties dans toute l'aire naturelle du sapin, de façon à couvrir toute la diversité des situations (âge, altitude, sol, topographie, végétation...), mais sans préjuger de létat sanitaire apparent des arbres. Sur chaque placette, six arbres ont été choisis parmi les dominants et codominants, notés quant à l'état de leur feuillage et carottés à cœur. Ce sont donc près de 1200 carottes, soit environ 120000 largeurs de cernes qui ont servi de base à l'étude dendrochronologique. Celle-ci a été alourdie par la fréquence élevée de «cernes manquants ". qu'il est impératif de déceler sous peine de désynchroniser toute la série de cernes antérieure. Cette phase délicate ("interdatation») est rendue possible grâce à la mise en évidence progressive d'années caractéristiques (quant à l'épaisseur ou à la minceur du cerne correspondant), telles $1870,1893,1916,1922,1934,1948,1956,1961,1976 \ldots$

Nous avons cherché à tester l'hypothèse selon laquelle l'élévation du niveau de pollution aurait entrainé, depuis une date à préciser, une baisse de vitalité de la sapinière, laquelle pourrait évoluer, depuis quelques années, en véritable dépérissement. De la masse des données disponibles, on a donc extrait tous les cernes élaborés à un âge courant fixé, mais, compte tenu de la large variété des âges actuels des peuplements, à des dates très variées. De façon aussi surprenante que nette. et quel que soit l'âge de référence choisi ( 60 à 110 ans), on ne constate aucune tendance générale significative dans l'évolution de la croissance radiale depuis le début du siècle... Dès ce stade de l'interprétation, il était donc possible d'affirmer que, contrairement aux craintes très vives qui prévalaient au moment où fut lancée l'étude, la sapinière vosgienne n'était pas menacée de disparition à très brève échéance.

Ceci n'excluait pourtant pas que cette réalité moyenne puisse cacher des situations particulières plus alarmantes et susceptibles de gagner ultérieurement en surface. Pour étudier cette éventualité, l'échantillon initial de placettes a été "stratifié " en un certain nombre de sousensembles selon la valeur de certains paramètres : altitude, fertilité minérale, exposition, position topographique. Auparavant, toutes les données ont été transformées en indices de croissance dégagés de l'influence de l'àge courant. Cette transformation s'est faite par référence à la courbe moyenne "largeur de cerne/âge " établic pour l'ensemble du dispositif. De façon troublante mais irréfutable, aucun des paramètres étudiés ne permet de discriminer des populations d'arbres ayant un comportement significativement différent de la moyenne. Par contre, toutes les courbes obtenues révèlent que la sapinière vosgienne a traversé effectivement une crise grave, amorcée dès 1973, ayant trouvé son paroxysme en 1976, mais pratiquement résorbée dès $1983 \ldots$ (du moins au vu du critère croissance radiale). Les mêmes courbes révèlent d'ailleurs d'autres crises comparables en ampleur et en intensité dans le passé : 1943-1951, "centrée"sur 1948, et 1917-1925, centrée sur 1922. Comme pour 1976, il y correspond à chaque fois des années ou séquences d'années à déficit pluviométrique marqué.

Il apparait de plus que l'aspect des houppiers et le taux apprécié de manque d'aiguilles ne sont que de médiocres indicateurs de la vitalité réelle des arbres: ce n'est qu'à partir de 40 p. 100 
environ que les courbes de croissance divergent significativement de la courbe moyenne. Par contre, la largeur d'aubier est un critère beaucoup plus pertinent : d'une part elle est très corrélée à la vigueur actuelle des arbres, d'autre part les courbes de croissance radiale des populations d'arbres correspondantes divergent depuis 50 à 60 ans, pratiquement depuis la crise climatique de 1917-1925.

Sans exclure totalement le rôle aggravant possible mais non démontré de la pollution à longue distance, il s'avère que les facteurs primaires de la crise récente, comme d'autres plus anciennes, traversée par la sapinière vosgienne, sont essentiellement d'ordre climatique. La diversité de réponse des arbres semble surtout liée à la structure des peuplements.

Mots clés : Dépérissement, dendrochronologie. climat. Abies alba, Vosges.

\section{Introduction}

Le « déclin ", le « dépérissement ", voire la «mort de la forêt », constituent des thèmes graves qui, depuis quelques années, préoccupent au plus haut point chercheurs et responsables forestiers dans un nombre croissant de pays, tant européens que nordaméricains. Malgré des études fort nombreuses, dont la synthèse devient de plus en plus complexe à mener (McLaughlin et al., 1983 ; Hermeline, 1984 ; Morrison, 1984 ; Schutt \& Cowling, 1985 ; Mclaughlin, 1985 ; Dessureault, 1985 ; Hinrichsen, 1986), force est de constater que les certitudes sont encore rares et que nombre d'interrogations essentielles demeurent.

Devant la multitude des hypothèses avancées, dont aucune ne s'avère jusqu'ici pleinement satisfaisante, il est de plus en plus tentant de parler de «complexe de causes ", dont la nature précise pourrait varier selon les régions, les époques et les espèces concernées. Parmi les causes les plus souvent évoquées figurent essentiellement la pollution atmosphérique, sous des formes extrêmement diverses - y compris à travers ses conséquences sur les propriétés de sols -, les accidents climatiques, les maladies, et, à un moindre degré, les erreurs sylvicoles.

Avec un léger décalage de quelques années par rapport à l'Europe moyenne, la forêt française paraît ne pas devoir faire exception au phénomène, et de nombreuses études, dont celle présentée ici, ont été engagées dans le cadre du vaste programme national DEFORPA (Dépérissement des Forêts attribué à la Pollution atmosphérique).

Il est utile de rappeler qu'au départ de notre étude (1983), les prévisions les plus alarmantes circulaient dans le monde scientifique - principalement en Allemagne - , reprises et souvent amplifiées par les medias : la forêt vosgienne était condamnée à très court terme ( 3 à 4 ans); certains symptômes laissaient même présager, dans les 10 années à venir, le déclin et la disparition des forêts européennes jusqu'à la pointe de la Bretagne. Et le scepticisme n'était alors guère de bon ton...

A notre point de vue d'écologiste forestier, il était indispensable, parallèlement aux études très spécialisées privilégiant tel ou tel type d'agression, de faire un point aussi objectif que possible sur la situation. Cette action devait selon nous avoir une assise statistique irréfutable, c'est-à-dire reposer sur l'analyse d'un grand nombre de situations, dans des conditions physiques et sylvicoles variées et connues, faire appel à des critères laissant un minimum de part aux notations subjectives, et - très important - donner une vision temporelle et dynamique des phénomènes observés.

Une étude phytoécologique et forestière classique, du type de celles menées pour les études de stations, associée à une étude dendrochronologique approfondie, nous 
paraissait pouvoir répondre à ces objectifs. On peut alors parler de dendroécologie, vocable de plus en plus usité en Europe et en Amérique du Nord.

\section{Domaine d'étude et techniques mises en euvre}

\subsection{Le choix des placettes d'étude}

Le choix de la région - le massif vosgien - et de l'essence - le sapin, Abies alba Miller - s'est imposé à nous : d'une part c'est sur eux que pesaient à l'origine les craintes les plus vives, d'autre part nous disposions déjà d'un réseau de 196 placettes mis en place peu avant pour étudier le déterminisme de la productivité du sapin.

Défini avant l'émergence de la problématique dépérissement, cet échantillon est donc parfaitement neutre de ce point de vue. Les placettes sont réparties sur l'ensemble de l'aire de l'espèce dans le massif vosgien, depuis la latitude de Saverne au Nord jusqu'à celle du Ballon d'Alsace au Sud, tant sur le versant lorrain que sur le versant alsacien. Leur choix visait à couvrir toute la diversité des situations possibles : géographique, géologique, altitudinale, topographique, contexte floristique, structure et âge des peuplements.

\subsection{Les observations phytoécologiques}

Elles comportent un relevé de végétation complet ainsi que les observations stationnelles classiques : altitude, pente, exposition, position topographique, humus, roche-mère, description de sol sommaire. Elles se répartissent comme suit :

\begin{tabular}{|c|c|c|}
\hline & Versant lorrain & Versant alsacien \\
\hline Nombre de placettes & 92 & 104 \\
\hline Age moyen $\ldots .$. & 98 ans (60 à 145 ans) & 96 ans (50 à 180 ans) \\
\hline Altitude moyenne & $585 \mathrm{~m}(280$ à $1070 \mathrm{~m})$ & $640 \mathrm{~m}(240$ à $1020 \mathrm{~m})$ \\
\hline
\end{tabular}

Les données floristiques ont été soumises à une analyse factorielle des correspondances (135 espèces analysées). Le plan factoriel $1 \times 2$ obtenu exprime la large prédominance d'un facteur responsable de la distribution des espèces, lequel a été interprété sans ambiguité comme un gradient d'acidité. Selon une technique maintenant éprouvée (KEENAN \& CANDY, 1983 ; BECKER, à paraître), ceci a permis de définir pour chaque placette un indice de nutrition minérale $N u$, qui est tout simplement la coordonnée de la placette sur l'axe 1 de l'analyse.

\subsection{Les observations sur les arbres}

Sur chaque placette, six arbres ont été choisis parmi les dominants et codominants, sans tenir aucun compte de leur état sanitaire apparent. Puis chacun a fait l'objet d'une 
notation visuelle sur l'état du feuillage, selon le même protocole que celui en vigueur sur les transects d'observation régulière du réseau DEFORPA. Nous l'appelerons It (indice de "transparence des houppiers", ou de "pertes d'aiguilles" ou de "manque d'aiguilles ») et préférons l'exprimer en pourcentage pour ne pas préjuger des classes traduisant le plus efficacement la vitalité des arbres.

Pour l'étude dendrochronologique proprement dite, une carotte de $5 \mathrm{~mm}$ de diamètre a été prélevée à cœur dans chacun des rnêmes arbres, à l'aide d'une tarière de Pressler. Le carottage a été fait à $1.30 \mathrm{~m}$ de hauteur, dans la direction des courbes de niveau du terrain, afin d'éviter le bois de compression ; méplats, boursouflures et autres anomalies géométriques du tronc ont également été écartés.

Les 1176 échantillons ainsi récoltés ont été soigneusement planés, au tiers environ de leur diamètre, à l'aide d'un rasoir histologique. La taille se fait après réhumidification de la carotte et dans un plan perpendiculaire au fil du bois. Toutes les largeurs de cernes (environ 110000 au total) ont été mesurées au dixième de $\mathrm{mm}$ près à l'aide de la machine d'Eklund de l'INRA ${ }^{(1)}$. Après stockage sur un support informatique, est venue la délicate phase dite d'interdatation, qui consiste à s'assurer très minutieusement du parfait calage chronologique de l'ensemble des largeurs mesurées, en déjouant tous les risques d'erreurs : erreurs de mesure, erreurs d'encodage, éventuels faux-cernes, et surtout cernes non mesurés, soit très fins et difficilement perceptibles, soit purement et simplement manquants. L'interdatation s'est faite graphiquement et grâce à la mise en évidence progressive d'un certain nombre d'années ou séquences d'années caractéristiques (le plus souvent marquées par une réduction brutale de la croissance radiale). Citons : 1870, 1890 à 1893, 1907, 1912, 1915-1916, 1922, 1929, 1934, 1948, 1956, 1961 , 1974 à 1976. Signalons que cette opération fondamentale s'est faite sur un écran de microordinateur et a été très largement facilitée par l'utilisation d'un programme permettant de faire défiler horizontalement, dans les deux sens, le profil dendrochronologique; ce programme offre également la possibilité de modifier instantanément l'échelle des dates, afin de débusquer plus commodément les cernes manquants (ou les faux cernes).

Sur les mêmes carottes a été mesurée la largeur d'aubier, qui s'avère souvent un excellent indice de vigueur de l'arbre (Waring et al., 1980) Hinckley \& Lassoie, 1981 ; Duhamel, 1984). Après tâtonnement, c’est finalement un trempage rapide dans une solution d'acide perchlorique à 40 p. 100 (Kutscha \& Sachs, 1962) qui se révèle le plus efficace pour le sapin, même sur carotte prélevée depuis plusieurs mois : après une trentaine de secondes, le bois de cœur se colore en vert. La différenciation cour-aubier est fugace ; la coloration verte, puis brune de plus en plus foncée, envahit ensuite toute la carotte ; le mieux est de revenir à l'état initial par un trempage dans une solution basique.

\section{Résultats}

L'interprétation des données disponibles s'est opérée en plusieurs étapes, selon une démarche qui n'était que très schématiquement esquissée à l'avance. En fait, chaque résultat partiel a très largement orienté le déroulement méthodologique de l'étape

(1) Misc aimablement à notre disposition par J. Bouchon. Station de Sylviculture et de Production. 
ultérieure. Le fil conducteur est le suivant : la sapinière est-elle significativement dans une situation grave ?; depuis quand ? est-ce un phénomène inédit ?; s'agit-il d'un phénomène général ou est-il limité à certaines situations particulières ?; y a-t-il des conditions aggravantes? Le rôle possible de la pollution est naturellement partout en filigrane... encore qu'une telle étude ne puisse, par nature, qu'apporter des présomptions plus ou moins convaincantes sur ce rôle.

\subsection{L'état des houppiers}

Rappelons qu'avant d'entreprendre l'étude dendroécologique, nous avons cherché à établir les corrélations entre l'état actuel des houppiers - en fait l'indice $I t$ - et les divers paramètres phytoécologiques disponibles (BECKER, 1985). Très succinctement, des techniques de régression progressive et d'analyse discriminante pas à pas avaient mis en évidence un certain nombre de grandes tendances :

— un effet défavorable de l'âge et de l'altitude ;

- sur le versant lorrain seulement, une sorte d'effet protecteur, pour une altitude et une latitude données, d'une ligne de crête élevée (la ligne de crête principale des Vosges, orientée nord-sud) ;

- une absence remarquable d'effet propre des autres facteurs stationnels, en particulier du niveau de nutrition minérale $N u$, alors que ce dernier intervient très significativement dans l'explication de la productivité des mêmes peuplements.

A ce stade de l'étude, les résultats, en particulier la dissymétrie de comportement des deux versants vosgiens, nous apparaissaient cohérents, au moins pour partie, avec l'hypothèse d'une pollution véhiculée par des vents d'est. Mais le classique effet de foehn qui caractérise le versant alsacien pouvait aussi être incriminé.

\subsection{Les difficultés d'interdatation chez le sapin}

Si la présence de doubles cernes s'avère pratiquement exclue (quelques rares cas douteux ont été écartés par une observation plus attentive), l'existence et la fréquence de séries plus ou moins longues de véritables « cernes manquants » ont singulièrement alourdi l'interdatation, et ceci malgré le statut social des arbres choisis :

1 cerne manquant et plus ............... 31 p. 100 des arbres

5 cernes manquants et plus . . . . . . . . . . . . . 12 p. 100 des arbres

10 cernes manquants et plus ................ 5 p. 100 des arbres

15 cernes manquants et plus . . . . . . . . . . . . 1 p. 100 des arbres

De telles difficultés avaient déjà été soulignées à propos de l'épicéa (VıNS, 1976). Ces chiffres méritent d'être présents à l'esprit pour les études dans lesquelles on est tenté de rapporter les $\mathrm{n}$ derniers cernes mesurés aux $\mathrm{n}$ dernières années... Pour tempérer la portée de cette observation, il convient cependant de la compléter en indiquant que ces cernes manquants s'insèrent en général dans des séquences de cernes de toute façon 1 rès étroits. Ces dernières sont également très fréquentes, à des périodes variées (mais surtout à partir de 1970) et correspondent à ce que ScHWEINGRUBER et al. (1983, 1985) appellent des " réductions abruptes de croissance ». Nous y reviendrons. 


\subsection{Appréciation globale de la vitalité de la sapinière vosgienne}

\subsection{Ce qu'il ne faut pas faire}

Une première tentation - à laquelle ont succombé de nombreux auteurs consiste à calculer la moyenne de tous les cernes disponibles à une date donnée et à construire la courbe donnant l'évolution de cette moyenne au cours des dernières dizaines d'années, sans autre précaution. Dans le cas de nos données, comme dans d'autres, on observe alors très "clairement" des fluctuations autour d'une valeur moyenne relativement constante depuis le début du siècle jusque vers 1940, suivies d'un fléchissement très marqué pouvant laisser craindre à bref délai l'éradication totale du sapin dans la région. Les autres dates de fléchissement souvent citées en Europe moyenne sont: les années 40, 1947-1953, 1950, les années 50, 1954, 1955, 1960, les années $60 \ldots$ De là à évoquer l'après-guerre, l'explosion de l'activité économique, l'élévation concomitante du niveau général de la pollution, il n'y avait qu'un pas qui fut souvent franchi.

Aucune conclusion solide, en fait, ne peut en être tirée, en raison :

- d'une part du vieillissement progressif de la population d'arbres correspondant à chaque date (lequel se traduit par une réduction naturelle de la largeur des cernes);

- d'autre part du nombre variable d'arbres disponibles à chaque date (nombre qui, dans notre cas, diminue rapidement avant 1945 (date d'installation des peuplements étudiés les plus jeunes).

\subsection{Croissance radiale à un âge de référence courant donné}

Une autre façon d'aborder la question fondamentale posée est la suivante : les arbres ayant 70 ans (par exemple) aujourd hui poussent-ils aussi bien, globalement, que leurs aînés lorsqu'ils avaient cet âge de référence de 70 ans ?

Pour répondre à cette question de façon rigoureuse, il est indispensable de disposer d'une très grande quantité de données, et que celles-ci portent sur des arbres d'âges aussi variés que possible, conditions sensiblement remplies dans la présente étude. On a donc extrait du fichier tous les cernes $L 70$ élaborés lorsque l'arbre avait cet âge de référence ( \pm 2 ans, pour amortir l'effet d'années très particulières), ainsi que les dates correspondantes. De la même façon, on a extrait tous les $L 50, L 60, L 80, L 90$ et $L I 00$, puis calculé, pour chaque lot de données, les moyennes aux différentes dates depuis le début du siècle. Malgré la taille du fichier disponible, la démarche utilisée fait que chacune de ces moyennes ne porte que sur un nombre $n$ de valeurs compris entre 1 et une quarantaine au maximum. La figure 1 ne conserve que les points correspondant à $n>4$.

L'observation de cette figure impose cependant une conclusion surprenante : on n'observe pas de tendance marquée dans l'évolution de la croissance radiale moyenne du sapin dans les Vosges depuis le début du siècle, et en tout cas pas la chute actuelle souvent avancée.

Qui plus est, une pente générale légèrement négative n'aurait même rien eu d'inquiétant. En effet, les arbres vieux échantillonnés aujourd'hui n'était probablement pas parmi les moins vigoureux dans la population totale des arbres du début du siècle. dont beaucoup ont disparu depuis. En revanche, l'échantillon actuel comporte aussi des 

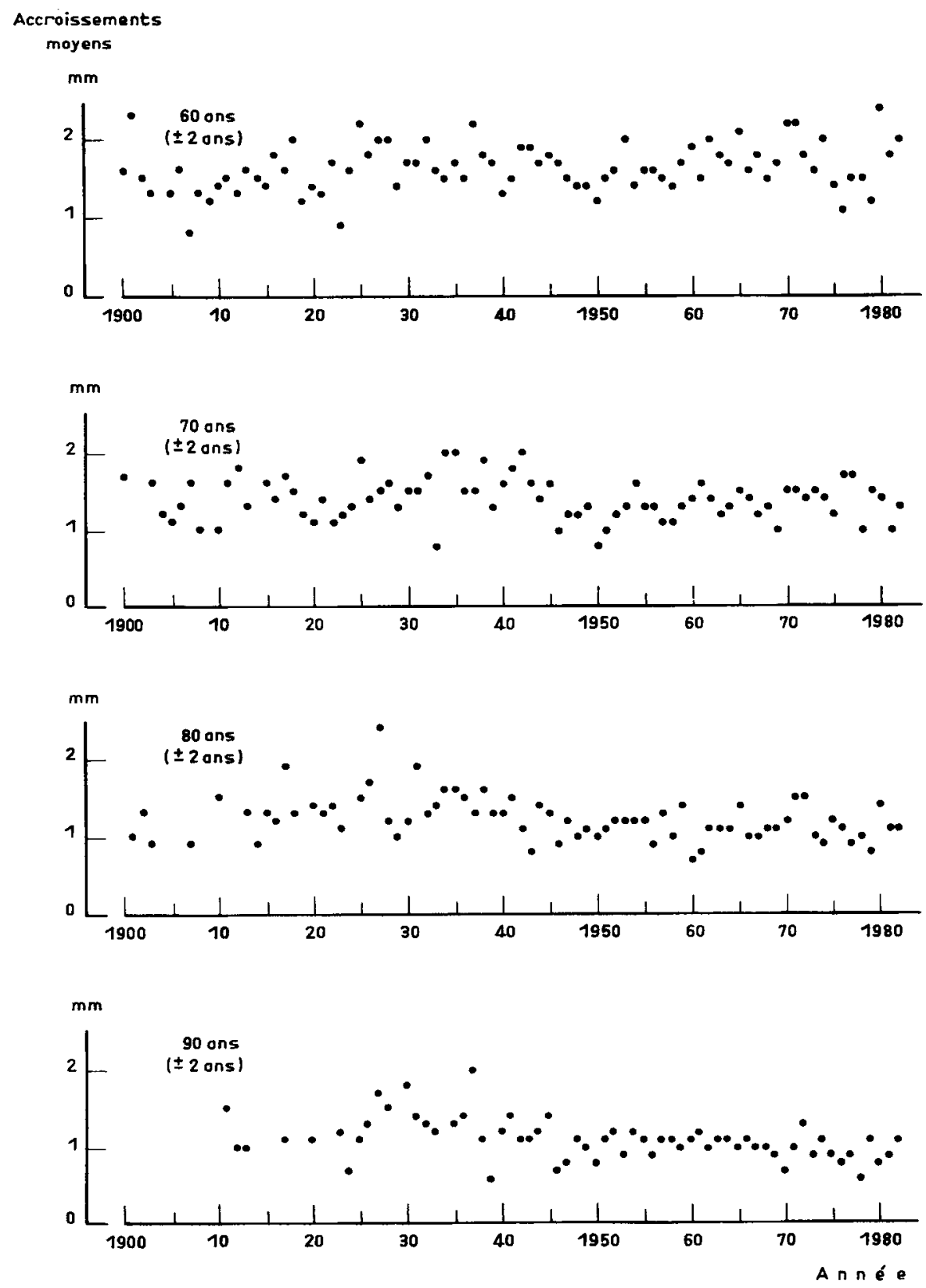

Fig. 1

Variation des accroissements radiaux moyens observés à différents àges courants de référence.

Variations in the mean ring widthes at various instant ages of reference. 
arbres peu vigoureux qui seront amenés à disparaître naturellement dans les années et dizaines d'années à venir.

A ce stade de l'interprétation, il est donc permis d'affirmer que, contrairement aux vives craintes exprimées à l'origine de l'étude, la sapinière vosgienne n'est pas, globalement tout au moins, dans une phase de clépérissement inéluctable ayant débuté il y a plusieurs décennies conjointement à l'élévation du niveau de pollution atmosphérique.

\subsection{Définition d'un indice de croissance indépendant de l'âge courant}

Il apparaît donc évident que l'âge courant perturbe beaucoup l'interprétation des largeurs de cernes. La figure 2, élaborée pour la classe d'âge (âge actuel) la plus fournie ( $80 \pm 2$ ans, 99 arbres) en est l'illustration. Si l'on fait abstraction des variations inter-annuelles rapides, on observe une tendance générale à la baisse très marquée dès le plus jeune âge.

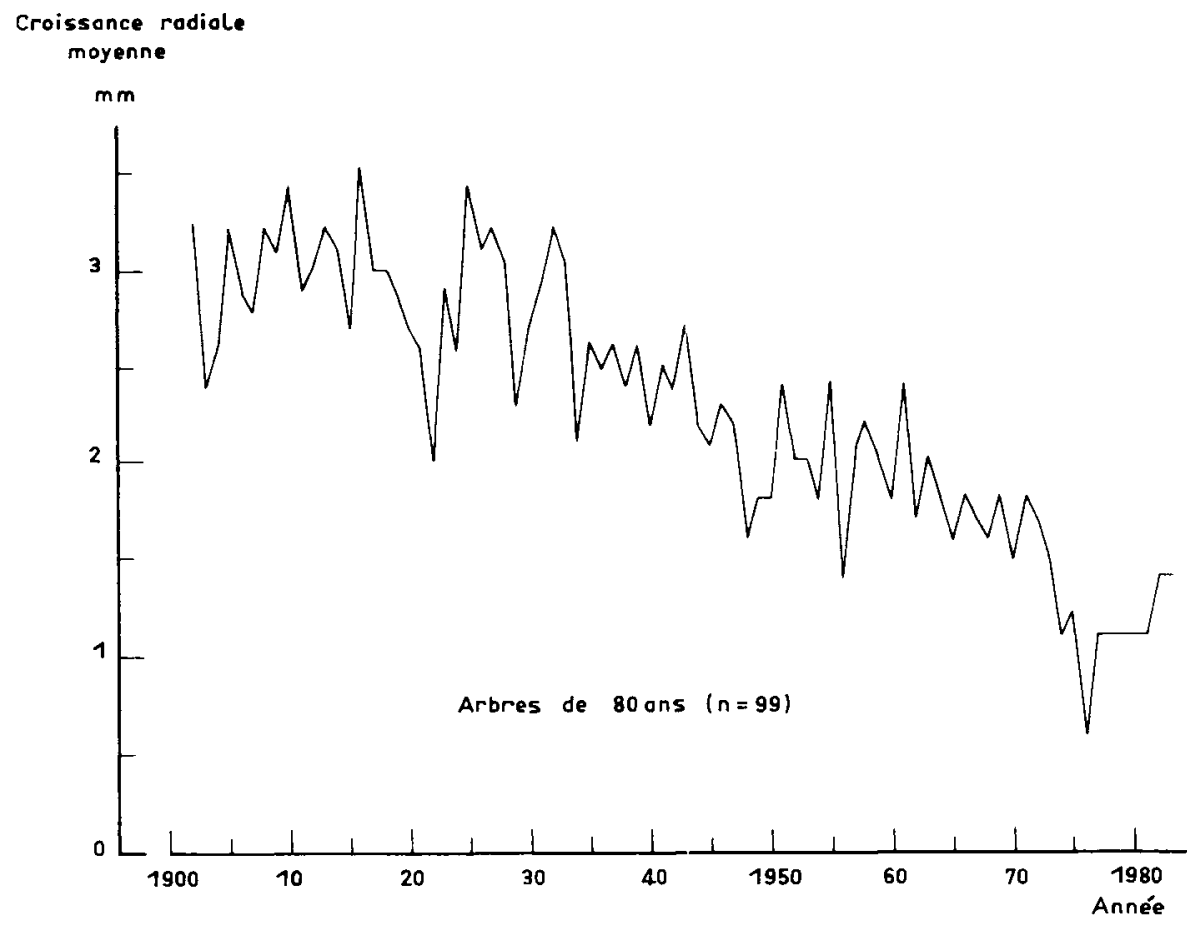

FIG. 2

Evolution dans le temps de la croissance radiale moyenne des arbres de 80 ans ( \pm 2 ans ; agge actuel).

Evolution according to calendar year of the mean radial growth of the 80 years old trees (土 2 years; present age).

Nous avons ensuite construit la courbe (fig. 3) donnant, pour des âges courants croissants, la moyenne de toutes les largeurs de cernes disponibles correspondantes (108 423 au total...). On constate que celle-ci, sans aucun «artifice » mathématique, 


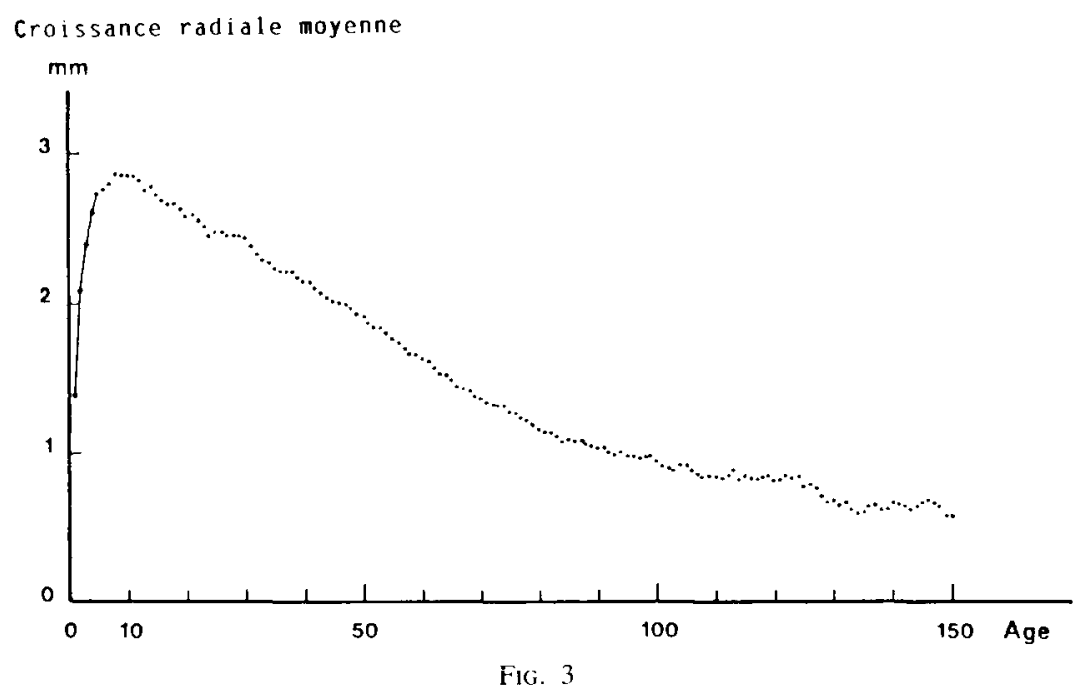

Evolution moyenne de la croissance radiale du sapin selon l'âge courant. Evolution according to instant age of the mean radial growth of white fir.

apparaît très régulière. Ceci tient, d’une part au grand nombre de données utilisées, d'autre part à la large diversité dans l'âge des arbres: de cette façon, à chaque âge courant de la courbe correspondent des dates d'élaboration des cernes, donc des conditions météorologiques, très variables, dont les effets contraires sur la croissance s'annulent statistiquement. La courbe obtenue, dont l'allure générale est d'ailleurs classique en dendrométrie (FrITTS, 1976), traduit donc la loi biologique de l'évolution moyenne de la croissance radiale du sapin dans les Vosges en fonction de leur âge, que nous appelerons $\mathrm{cm}(\mathrm{a})$.

Dans l'étape suivante, chaque largeur de cerne observée à un âge courant $a$, soit co (a), est transformée en un «indice de croissance » $i$ (a) (McLaughlin et al., 1983 ; Serre-BACHet, 1982), qui permet ensuite de comparer chaque valeur obtenue indépendamment de l'âge d'élaboration :

$$
i(a)=100 \cdot c o(a) / \mathrm{cm}(a)
$$

On peut alors revenir à la date et construire la courbe donnant l'évolution de l'indice de croissance $i(d)$ depuis le début du siècle (fig. 4). Elle confirme largement, sur une base statistique beaucoup plus large, les résultats livrés par la figure 1.

Elle laisse cependant deviner l'existence d'un optimum dans les années 30 . La même courbe, mais établie depuis 1850 , renforce très nettement le phénomène : globalement le niveau de croissance radiale du sapin dans les Vosges a augmenté d'environ 70 p. 100 entre 1850 et 1935 , pour baisser ensuite légèrement de 10 à 15 p. 100. Ce résultat, qui a peut-être une portée dépassant largement le cadre géographique étudié, est analysé par ailleurs (BECKER, 1987). Elévation du taux de $\mathrm{CO}_{2}$ atmosphérique et, surtout, variations lentes de la température au niveau mondial et de la pluviométrie correspondante semblent les responsables les plus probables. Quoi qu'il en soit, contrairement à certaines craintes, la croissance de la sapinière est en moyenne supérieure actuellement à ce qu'elle était au siècle dernier... 


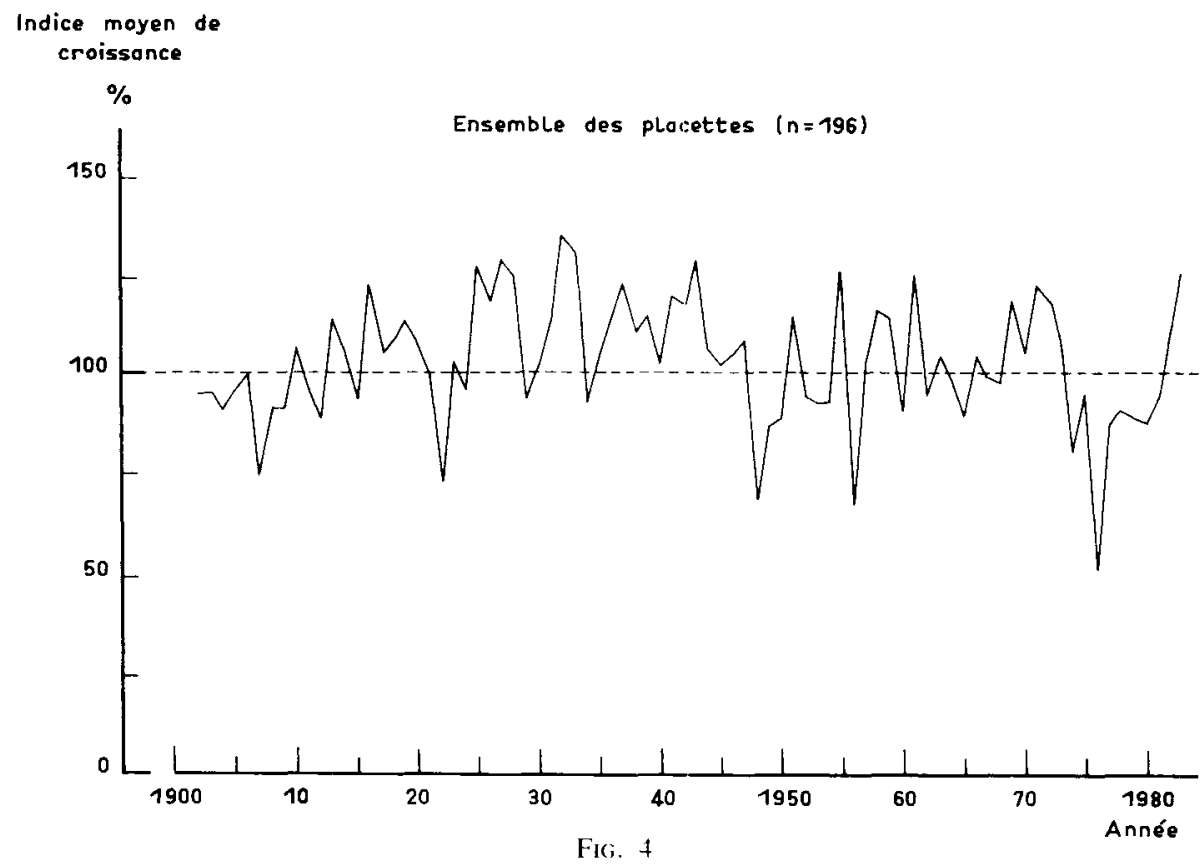

Evolution dans le temps de l'indice moyen de croissance radiale sur l'ensemble des placettes. Evolution according to calendar year of the mean radial growth index for all of the stands.

\subsection{Les crises de la sapinière au cours du $X X^{r}$ siècle}

L'observation plus détaillée de la figure 4 livre d'autres informations importantes. La crise « actuelle » de la sapinière y est très visible et profonde ; mais elle débute en fait dès 1973, trouve son paroxysme en 1976, et, surtout, on observe un net rétablissement à partir de 1981. Des données partielles, non présentées ici, montrent que l'amélioration s'est fortement confirmée en 1984,1985 et 1986.

Cette crise, que l'on pouvait déjà remarquer sur la figure 2 , s'avère non unique au cours de ce siècle. On peut en relever au moins deux autres, presque comparables en durée et en intensité : 1916-1925, centrée sur 1922, et 1943-1951, centrée sur 1948. II est d'ailleurs permis de penser qu'elles ont été en fait plus profondes encore qu'il n’y paraît, car nombre d'arbres les plus touchés ont dû disparaître depuis. Seule la seconde semble avoir été rapportée dans la littérature française : ainsi Fourchy (1951) décrit-il des symptômes et aspects de peuplements qui étaient tout à fait d'actualité en 19831984... La littérature allemande, analysée par CRAmer (1984), est plus prolixe et relate parfaitement les deux crises, ainsi que d'autres événements plus anciens encore, mais sans être très unanime sur les causes. Il est remarquable de constater qu'un grave dépérissement du chêne a également sévi dans diverses régions françaises au cours de ces trois mêmes périodes (Turc, 1927 ; Rol. 1951 ; Becker \& Levy, 1983).

Le déterminisme de la croissance radiale sera étudié ultérieurement de façon plus approfondie. Les données météorologiques de Strasbourg (fig. 5) permettent cependant, 


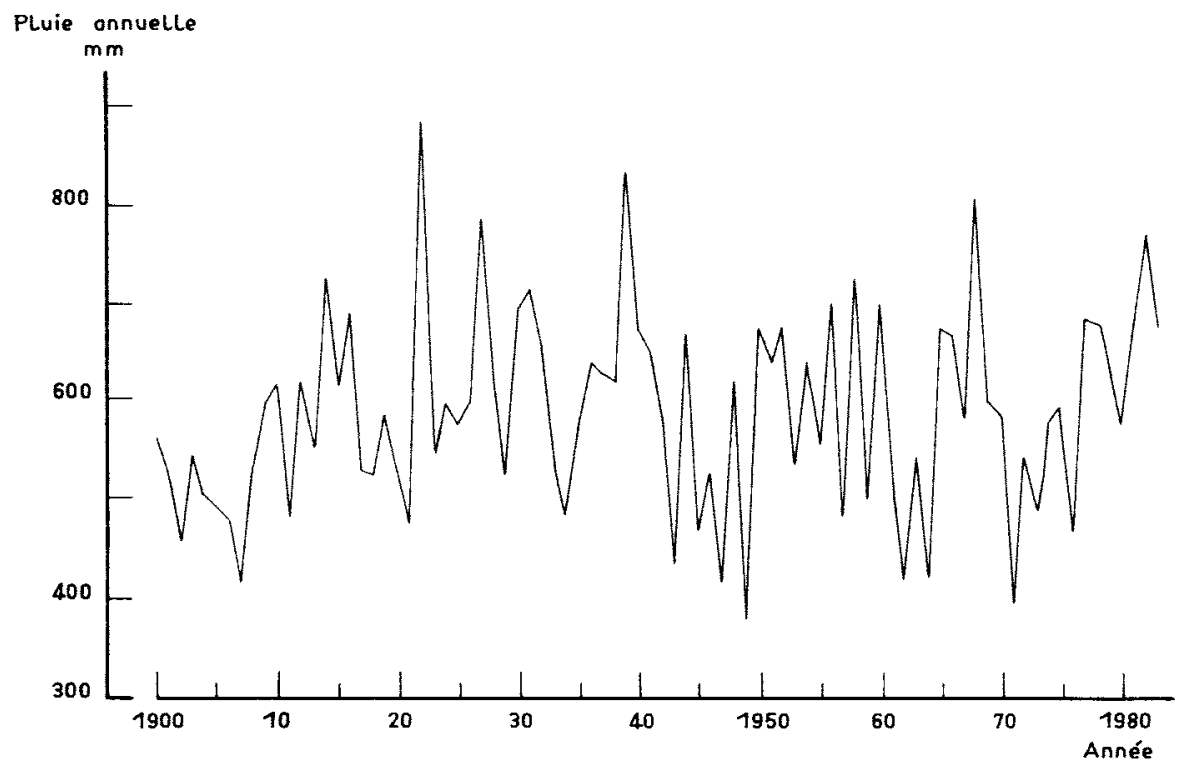

Pluie de

Mai à Septembre

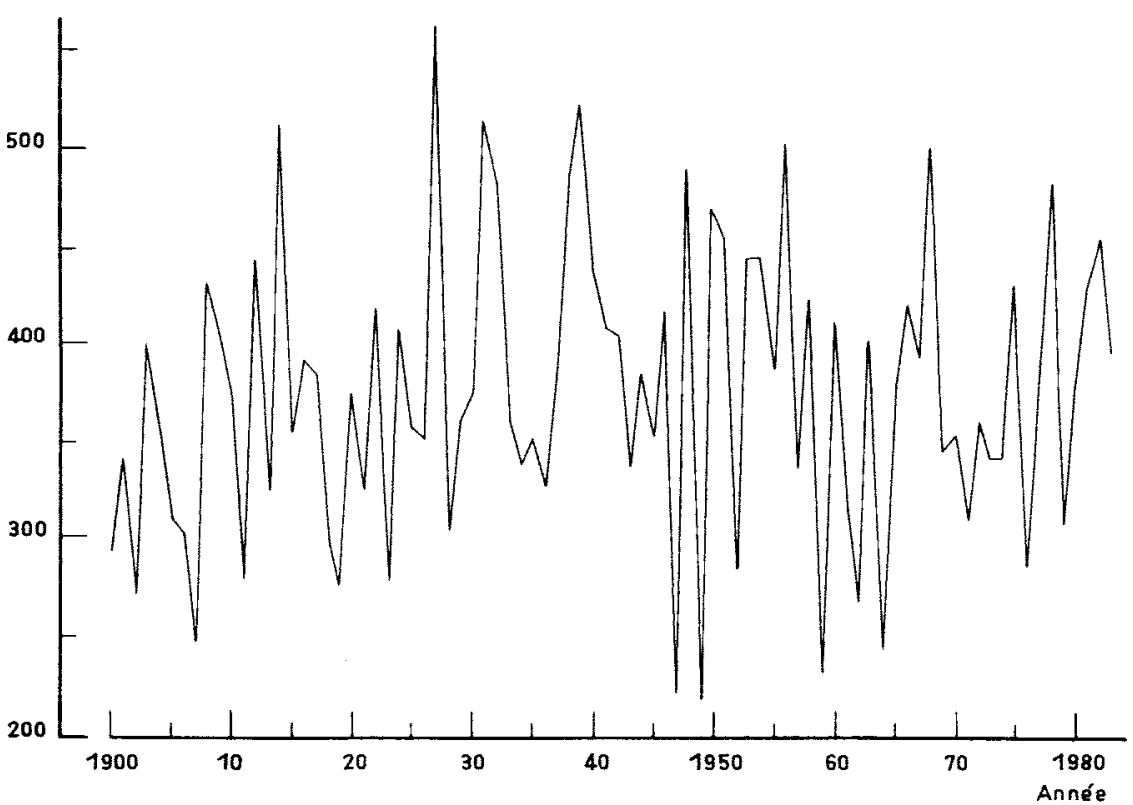

FIG. 5

Données pluviométriques au poste météorologique de Strasbourg-Entzheim. Totaux annuels et totaux de mai à septembre.

Rain amounts at Strasbourg-Entzheim (annual and may to september amounts). 
par la simple observation, de mettre en évidence le rôle décisif des périodes à déficit pluviométrique plus ou moins long et marqué sur le déclenchement des crises évoquées : 1917-1921, 1943-1949, 1969-1976. Des séquences plus courtes, telles 1928-1929 et 1933-1934, ont également une répercussion très riette sur la croissance radiale. Seule la séquence 1961-1964 n'a eu globalement qu’un€: incidence limitée sur la courbe de croissance ; mais une étude complémentaire (LEvy \& BEckER, 1987) montre son rôle décisif pour expliquer la situation actuelle des peuplements aujourd'hui encore nettement dépérissants. Remarquons qu'une série de séquences déficitaires telle que celle vécue depuis quarante ans - période courte dans la vie d'un écosystème forestier est unique depuis que l'on dispose de données météorologiques régulières (1880 à Strasbourg). Il n'est donc pas surprenant a priori que la crise actuelle puisse être également tout à fait exceptionnelle...

\subsection{Stratification des données selon le: caractéristiques stationnelles}

Les résultats déjà présentés permettent donc d'écarter l'hypothèse du cataclysme prédit il y a quelques années, et même celle d'un désordre profond et durable affectant l'ensemble de la sapinière vosgienne. Mais rien n'empêche d'imaginer que cette réponse globale masque d'importantes différences locales, et que certaines situations particulières très touchées puissent préfigurer, après une évolution à définir, le sort d'une surface toujours croissante de sapinières.

\subsection{Altitude}

L'échantillon de placettes a été réparti en 7 strates selon leur altitude (effectif de chaque strate compris entre 102 et 252 arbres). Puis 7 courbes du type de celle de la figure 4 ont été construites. Alors que l'aspect des houppiers (indice $I t$ ) était apparu très lié statistiquement à l'altitude (BECKER, 1985), on ne relève ici aucune différence significative entre ces courbes, qui se superposent de façon pratiquement parfaite.

\subsection{Exposition}

Selon une technique exposée par ailleurs (BECKER, 1979), les données de pente et d'exposition ont été converties en un indice d'exposition $I r$ traduisant en fait le climat radiatif local. Les données furent stratifiées en 4 lots (effectifs compris entre 258 et 318 arbres). Là encore, on n'observe aucune différence significative entre les courbes d'indice de croissance correspondantes.

\subsection{Distance altitudinale à la crête principale}

Cette variable s'était également révélée utile dans l'explication de l'indice It. Il s'agit de la différence d'altitude entre la placette étudiée et la crête principale des Vosges à la même latitude. Sept courbes d'indice de croissance ont été établies (effectifs compris entre 48 et 420 arbres), avec le même résultat négatif que précédemment...

\subsection{Indice de nutrition minérale}

L indice $N u$, issu de l'analyse statistique des données floristiques, a permis de la même façon d'élaborer 5 courbes (effectifs : 42, i86, 216, 318 et 414 arbres). Quatre de ces courbes se superposent ici encore parfaitement. Seule celle correpondant aux 
milieux les plus acides $(n=42)$ se distingue : elle fluctue d'abord significativement en dessous des trois autres, s'y mélange ensuite pendant quelques dizaines d'années, puis passe assez nettement au dessus depuis une quarantaine d'années.

\subsection{Position topographique}

Quatre classes ont été notées sur le terrain : fond de vallon $(\mathrm{n}=30)$, bas de pente $(\mathrm{n}=204)$, mi-pente $(\mathrm{n}=552)$, haut de pente $(\mathrm{n}=390)$. Même résultat décevant : seule la courbe «fond de vallon fluctue longuement au-dessus des trois autres avant de les rejoindre.

En fait, ce résultat et le précédent (indice $\mathrm{Nu}$ ) sont seulement cohérents avec l'évolution normale de la croissance radiale au cours de la vie d'un peuplement: les différences de fertilité stationnelles s'expriment essentiellement au début, puis s'estompent graduellement (ABETZ, 1985).

\subsection{Latitude}

Par «curiosité », nous avons cherché à mettre en évidence un éventuel effet géographique sans relation étroite avec les variables précédentes. Pour chacun des deux versants vosgiens, quatre territoires ont été définis, du nord vers le sud. Globalement, on constate, en particulier sur le versant alsacien, que les courbes d'indices de croissance sont largement superposables jusque vers 1960 , puis qu'elles divergent significativement, les régions sud $(n=258$ arbres $)$ et centre-sud $(n=264)$ conservant une croissance supérieure à celle des régions nord $(n=312)$ et centre-nord $(n=342)$. Nous verrons plus loin, à la lumière dautres résulats, comment interpréter cette observation.

\subsection{L'état des houppiers et la vitalité réelle des arbres}

Nombre des résultats présentés jusqu'ici sont à première vue assez déconcertants, et doivent surtout leur crédibilité à la large assise statistique délibérément voulue à l'origine. Certains sont cohérents avec les premières conclusions tirées de la seule observation des houppiers, en particulier l'absence de relation entre symptômes du « dépérissement» et fertilité minérale globale des sols. Mais d'autres ne sont pas confirmés, et en tout premier lieu l'effet négatif de l'altitude.

Nous nous sommes donc interrogé sur la pertinence du critère « aspect des houppiers » pour juger de la vitalité réelle des arbres... Comme précédemment, nous avons donc construit une famille de courbes d'indices de croissance correspondant à autant de classes d'indice $I t$, de 5 p. 100 en 5 p. 100 . Le résultat est là encore surprenant: jusqu'à 35 p. 100 de manque d'aiguilles, on n'observe aucune différence significative. Au-delà seulement, la courbe diverge nettement de la précédente (fig. 6), et ceci, en moyenne, depuis une trentaine d'années environ.

L'aspect des houppiers — du moins tel qu'il est habituellement apprécié jusqu'ici - apparaît donc n'être qu'un médiocre indicateur de la vitalité réelle des sapins jugée d'après le critère croissance radiale à $1,30 \mathrm{~m}$. La localisation du carottage ne paraît pas introduire de biais; selon KIENNEN \& Schuck (1983), les sapins dépérissants réduisent en effet d'abord leur croissance à la base des troncs et non au sommet. A l'occasion du dépérissement du chêne, nous avions déjà mis en évidence le caractère souvent 


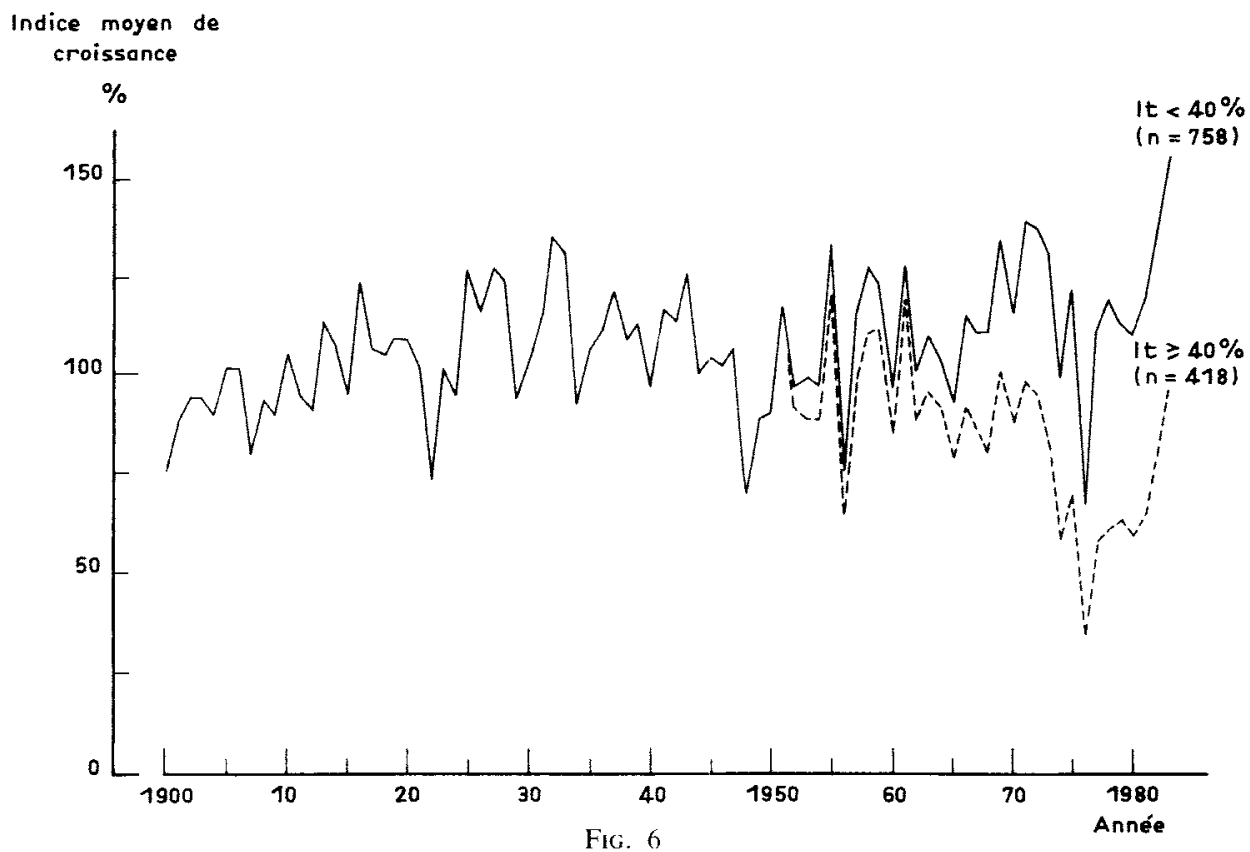

Evolution dans le temps de l'indice de croissance radiale selon la valeur de l'indice correspondant It de transparence des houppiers.

Evolution according to calendar year of the mean radial growth index. Influence of the it index of the corresponding trees (It : needle losses index).

trompeur de l'aspect des houppiers (Becker \& Levy, 1983). Cette difficulté vient d'être également soulignée par Pollanschutz (1985), Bauch et al. (1985), Kramer (1986) et EichKorn (1986) pour l'épicéa, ainsi que par Schweingruber (1983) pour le sapin.

\subsection{Intérêt du critère largeur l'aubier}

On sait que la section d'aubier est très corrélée - au moins hors périodes de crise - avec la surface foliaire totale de l'arbre (Hinckley \& Lassoie, 1981 ; Granier, 1981 ) ; elle a d'ailleurs été utilisée pour élaborer un "indice de vigueur » (WaRING et al., 1980).

Toujours selon le même principe que précédemment, nous avons élaboré une famille de courbes d'indices de croissance correspondant à diverses classes de largeur d'aubier. Auparavant, nous avons établi que celle-ci était dépendante de l'âge de l'arbre, selon la loi générale suivante :

$$
L a(\mathrm{~mm})=65 \cdot \operatorname{EXP}(-0,01 \cdot \text { âge })+35
$$

Toutes les largeurs d'aubier ont été converties en indices, comme pour l'obtention des indices de croissance. Cinq classes d'indices de largeur d'aubier ont été établies: $<40$ p. $100(\mathrm{n}=41$ arbres $), 40-79$ p. $100(\mathrm{n}=253), 80-119$ p. $100(\mathrm{n}=526), 120-159$ p. $100(\mathrm{n}=287), \geqslant 160$ p. $100(\mathrm{n}=59)$. 
La figure 7 est saisissante... On obtient un faisceau de courbes très nettement et constamment divergentes depuis une soixantaine d'années! Nous commenterons plus longuement ce résultat dans la discussion finale. Mais dès à présent on peut affirmer que la largeur d'aubier est, en période de crise au moins, un indice de vitalité des arbres très supérieur à l'indice de manque d'aiguilles. Il est malheureusement aussi plus. difficile à mesurer de façon très répétitive.

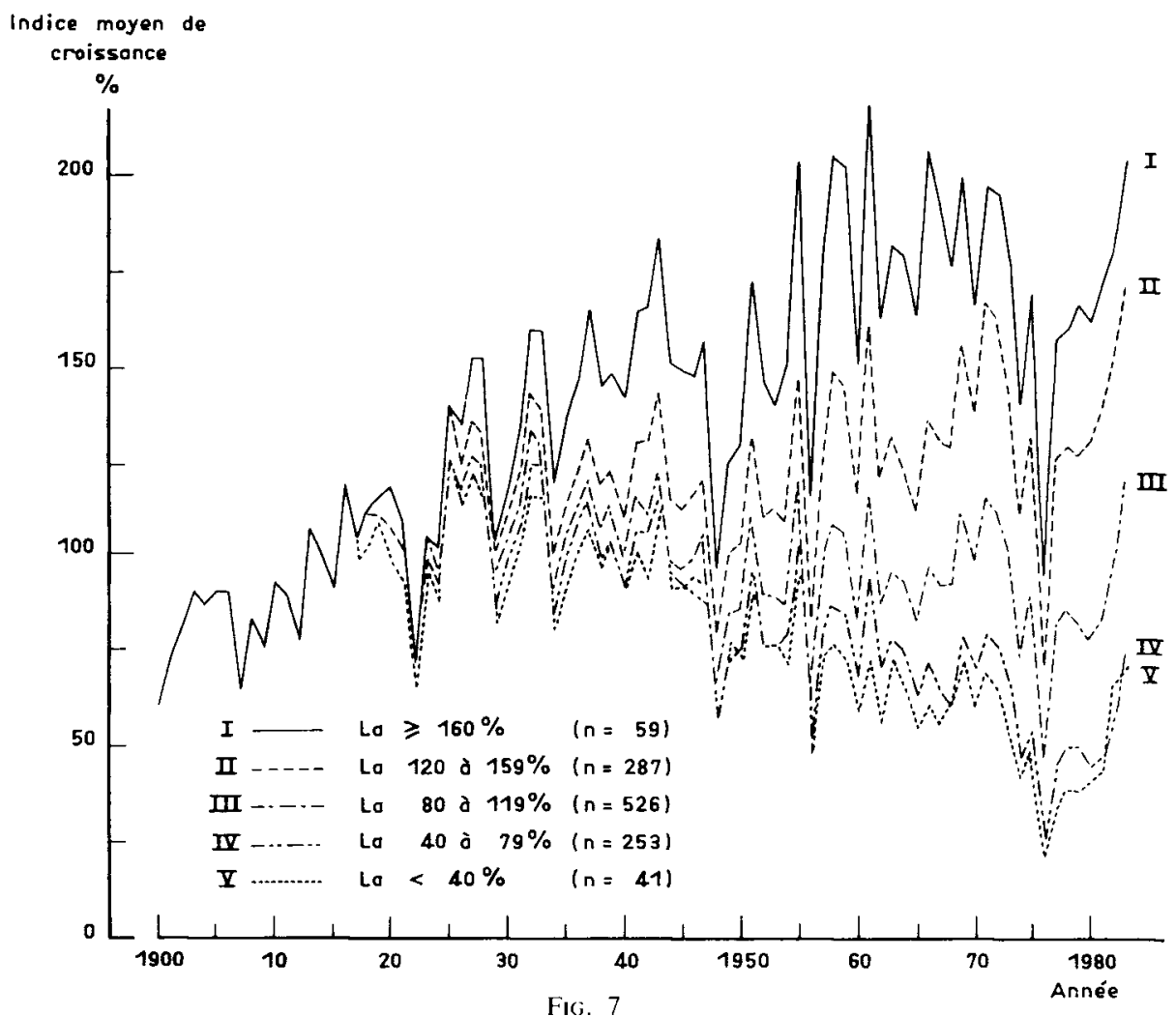

Evolution dans le temps de lindice de croissance radiale selon la valeur de lindice correspondant La de largeur daubier (corrigée selon l'àge de l'arbre).

Evolution according to calendar year of the mean radial growth index. Influence of the La index of the corresponding trees (La : sapwood width index).

Sur un plan plus fondamental, il en ressort que la chute d'aiguilles n'est pas systématiquement synonyme de baisse de production, ainsi que le fait remarquer aussi Pollanschutz (1985) à propos de l'épicéa (en situant le seuil sensible à $20 \mathrm{p} .100$ de pertes). Ce sont d’ailleurs les générations d’aiguilles les plus anciennes qui tombent, et l'on sait que leur efficacité photosynthétique est très inféricure à celle des plus jeunes aiguilles. 


\subsection{Définition et étude d'un indice de crise}

La courbe moyenne d’indice de croissance a été construite pour chacune des 196 placettes du dispositif ( 6 arbres par placette). Un examen attentif des 196 profils montre que la grande majorité des populations d'arbres étudiées ont bien "récupéré " après la crise des années 40 . Ceci n écarte bien sûr pas l'hypothèse que des arbres plus ou moins nombreux y aient disparu depuis, qui ne sont donc plus là pour témoigner... Ce n'est pas le cas pour la crise (beaucoup plus légère en moyenne) des années 60 . consécutive semble-t-il à la sécheresse des années 1962 et 1964.

Nous référant donc, pour chaque placette, au niveau d'indice de croissance moyen des années 50, nous avons défini un «indice de crise", qui est la surface comprise entre ce niveau et la courbe observée depuis, en ne prenant en compte que les portions de courbe situées sous le niveau de référence.

Nous avons soumis cette nouvelle variable à une régression multiple pas à pas faisant intervenir les variables explicatives déjà étudiées : altitude de la crête principale, latitude, indice de nutrition, indice d'exposition, position topographique ; et en ajoutant la proportion de hêtre dans le peuplement.

Là encore, aucune des variables écologiques strictement stationnelles n'intervient. Seule la latitude apparaît significativement corrélée à l'indice de crise (F partiel = 11,0). Et il s'agit bien d'un effet strictement géographique, car le remplacement forcé de la latitude par les variables qui lui sont le plus corrélées (altitude, altitude de la crête) provoque un effondrement des $F$ partiels. Ceci est d'ailleurs cohérent avec certaines observations précédentes (paragraphe 3.46).

Une vision cartographique des données confirme et éclaire ces résultats. Les placettes caractérisées par un indice de crise élevé se distribuent de façon sensiblement uniforme dans le massif vosgien, quelles que soient la roche-mère et la richesse des sols correspondants, sauf dans la partie sud - très schématiquement au sud du Col du Bonhomme - où elles sont très nettement moins nombreuses.

Or cette région présente effectivement certaines originalités générales. Les sols au moins dans l'aire du sapin - y sont pratiquernent toujours bien pourvus en bases (développés sur granites riches) ; mais de telles conditions se retrouvent également dans la moitié nord du massif. Par contre, le bioclimat y est spécifique (CaCHaN, 1974). En moyenne, la pluviométrie l'emporte nettement sur l'évapotranspiration potentielle $(\mathrm{P}-\mathrm{ETP}>100)$, alors que le bilan fluctue entre 100 et 0 , voire -100 , dans les régions sur granite riche de la moitié nord des Vosges.

Bien que non statistiquement significatif ( $F$ partiel $=2,3$ ), le rôle de la proportion de hêtre dans les peuplements intervient en deuxième position dans la régression progressive. Le rôle positif d'un meilleur équilibre des deux espèces n'est donc peutêtre pas à exclure. Rappelons que, climaciquemerit, la sapinière vosgienne est en fait une hêtraie-sapinière. 


\section{Discussion générale et perspectives}

\subsection{La sapinière vosgienne n'est pas menacée de disparition}

Au cours des toutes dernières années, divers auteurs, sur la base de travaux basés également sur l'analyse rétrospective de la croissance radiale, ont avancé l'hypothèse d'un déclin sensible du sapin et de l'épicéa en Europe moyenne et du nord depuis 20 à 40 ans (Aldinger \& Kremer, 1985 ; Athari, 1983 ; Berchtold et al., 1981 ; Kenk, 1983 ; Kenk et al. 1984 ; JonsSON \& SundBerg, 1972 ; Mitscherlich, 1983 ; MoosmAyer, 1984 ; Schweingruber et al.. 1983 ; Schweingruber, 1985). Nous ne confirmons pas cette crainte pour la sapinière vosgienne, et nous pensons que certains aspects méthodologiques peuvent expliquer cette divergence. En cela, nous rejoignons d'ailleurs d'autres travaux très récents, dont l'approche méthodologique nous paraît plus convaincante (Gerecke, 1986, sur le sapin : Thren, 1985, sur le pin sylvestre : Eichkorn, 1986. sur l'épicéa).

\subsection{Expliquer certaines contradictions apparentes}

Pour être plus convaincant - et convaincu nous-même - nous avons aussi cherché à comprendre les raisons profondes de certaines contradictions apparentes entre nos résultats actuels et diverses observations rapportées par ailleurs, et même certaines des nôtres au début de notre étude.

\subsection{L'aspect des houppiers}

Nous avons déjà débattu du caractère souvent trompeur de l'aspect des houppiers lorsque l'on doit apprécier visuellement l'état de vigueur d'un arbre, tout en reconnaissant qu'il s'agit là du seul critère vraiment utilisable à une vaste échelle... Il serait donc utile d'affiner, sans trop les alourdir, ces notations, à la recherche de critères plus pertinents. En attendant, il apparaît peu utile de trop s'attarder sur l'état d'un sapin tant que l'indice de manque d'aiguilles - tel qu'il est apprécié actuellement - ne dépasse pas $40 \mathrm{p} .100$ (correspondant à la partie la plus endommagée de la classe 3 de l'échelle DEFORPA, qui est parfois, par commodité, notée $3 b$ par certains auteurs). Nous avons déjà eu l'occasion de souligner le caractère impropre de l'échelle mise au point à l'origine par l'Allemagne et largement reprise par divers pays, en particulier de la note 3, beaucoup trop large $(20$ à 60 p. 100 en France ; subdivisée ensuite en 3a et 3b).

\subsection{Le rôle de la nutrition minérale}

Une étude détaillée menée dans les Vosges (Landmann et al., 1987) montre une corrélation significative, à l'intérieur de l'échantillon de placettes étudié, entre vigueur du sapin (critère visuel d'aspect des houppiers), richesse minérale du sol et statut nutritif actuel des arbres (analyses foliaires); certaines difficultés d'alimentation en magnésium sont en particulier soulignées. Or, dans aucune des multiples "tentatives" que nous avons faites, nous n'avons pu confirmer le rôle de la richesse minérale globale des sols - appréciée par la composition floristique - sur la vitalité des peuplements jugée sur le critère croissance radiale.

Certes la végétation ne rend peut-être pas compte spécifiquement de la nutrition magnésienne. Mais, après une étude dendroécologique complète des placettes retenues 
dans cette étude, il apparaît que la quasi-totalité des peuplements classés 3b - les seuls réellement en situation inquiétante - sont localisés sur des sols pauvres. Or nous avons vu que dans notre échantillon - beaucoup plus large et établi au hasard - la probabilité de crise est sensiblement la même partout (sauf dans le Sud des Vosges, doté d'un bioclimat plus humide).

Là encore, il semble que l'aspect des peuplements ait pu peser de façon excessive dans le choix des placettes. Ouoi qu'il en soit. la mise en place d'un échantillon complémentaire destiné aux analyses foliaires est programmé pour approfondir cette question fondamentale.

\subsection{Reprise de croissance et apparition du "dépérissement"}

Il est peut-être encore plus troublant de constater le net décalage - plusieurs années - entre le plus profond de la très réelle crise traversée par la sapinière vosgienne (1976) et le début des vives inquiétudes manifestées en France (1982). Or, dès cette date, la croissance radiale était en moyenne redevenue normale. Et depuis, en particulier en 1984 et 1985, l'étude de placettes complémentaires (LEVY \& BEcKER, 1987) montre qu'elle est même devenue très supérieure à la moyenne observée depuis un siècle... Il n'est pas impossible qu'un lessivage des feuillages par les dépôts acides ait également contribué au phénomène.

Plusieurs raisons peuvent expliquer ce paradoxe irritant. On ne sait malheureusement pratiquement pas quand ont réellement débuté les chutes d'aiguilles, ni à quelle vitesse ; sans doute bien avant que l'on ne s'en soit vraiment inquiété... Par ailleurs, rappelons une fois encore qu'il s'avère qu'une activité photosynthétique sensiblement normale peut avoir lieu avec un nombre de générations d'aiguilles inférieur à la moyenne.

Mais nous voudrions avancer ici une autre hypothèse quelque peu provoquante : l'état actuel préoccupant des arbres ne serait-il pas, dans certaines situations, non l'expression de leur dépérissement mais au contraire celle de leur regain de vigueur? Ceci vaut surtout pour les symptômes de jaunissement qui, bien que moins nettement que chez l'épicéa, accompagnent souvent celui du manque d'aiguilles.

Sur les sols pauvres chimiquement, la disponibilité des éléments minéraux est limitée. Or les conditions climatiques - pluviométriques surtout - de ces dernières années ont été très favorables à la croissance. Le stock des éléments disponibles a donc dû se «diluer» dans une masse végétale nouvelle plus importante, ceci s'extériorisant par des symptômes visuels et analytiques de carence... Il n'est pas impossible qu'un lessivage des feuillages par les dépôts acides ait également contribué au phénomène.

Cette hypothèse apporterait d'ailleurs un complément d'explication au problème d'échantillonnage soulevé précédemment (paragraphe 4.22). De tels symptômes de dilution ne peuvent en effet s'exprimer sur les sols où la disponibilité des cations est importante, que les peuplements aient ou non traversé une crise importante quelques années auparavant.

\subsection{La mort des arbres}

Bien que ce ne soit pas le trait dominant des phénomènes observés depuis quelques années, il faut cependant retenir que la mortalité des arbres a été plus élevée 
que par le passé ; et le vieillissement — réel — de la sapinière vosgienne ne suffit pas à justifier ce fait.

L'explication proposée est suggérée par l'observation de la figure 7 . Celle-ci nous paraît synthétiser toute la vie d'un écosystème forestier, qui - le sylviculteur le sait bien -- se traduit d'abord par une âpre compétition entre les individus : inéluctablement, parmi les quelques centaines de milliers de semis présents à l'hectare au moment de la régénération naturelle, seuls quelques centaines parviennent jusqu'au peuplement final qui assurera la génération suivante. Entre temps, que ce soit spontanément ou du fait de l'action du forestier, progressivement ou par à-coups, la très grande majorité des individus doit disparaître.

Sauf accident, ce sont les individus les moins vigoureux qui disparaissent. La figure 7 montre qu'en moyenne le statut social de dominé, traduit par la section d'aubier actuelle, est le résultat d'un processus très long (une soixantaine d'années dans l'échantillon étudié). En période "normale", la mortalité naturelle, sensiblement constante, est acceptée comme telle aussi. Que survienne une séquence climatique défavorable, telle celles des années $20,40,60$ ou 70 , et les conséquences de la compétition s'expriment alors plus brutalement par une mortalité « anormale », dont on peut alors s'inquiéter. L'espace vital ainsi rapidement libéré est d'ailleurs favorable à un regain d'autant plus important de la croissance des survivants une fois le stress climatique terminé. C'est ce que l'on observe actuellement.

\subsection{Conclusion et perspectives}

Le rôle des facteurs climatiques avait été largement éludé jusqu'ici dans la plupart des très nombreuses publications européennes sur le dépérissement des forêts. L'hypothèse sécheresse a cependant été avancée à plusieurs reprises, mais sans retenir suffisamment l'attention de la communauté scientifique: Alcubilla et al. (1985), Clauser (1981), Cramer (1984), Cramer \& Cramer-Middendorf (1984), Gerecke (1986), Kienast et al. (1981), Pollanschutz (1985), Thren (1985), Eichkorn (1986), Larsen (1986), SPIEcker (1986). Elle est évoquée, puis écartée par Schweingruber et al. $(1983,1985)$.

Au terme de la présente étude, il apparaît évident que les « accidents » climatiques - et tout spécialement les sécheresses - ont eu un rôle très important dans le déterminisme des crises traversées par la sapinière vosgienne. Nous ne pouvons bien sûr pas affirmer qu'ils soient exclusifs : la pollution générale - qu'il importe de toute façon de réduire coûte que coûte -- peut jouer actuellement un rôle aggravant, qu'il nous est impossible de confirmer ni d'infirmer.

Cette constatation générale sur le rôle du climat ne rend cependant pas compte de toutes les situations. Certains peuplements ont été globalement beaucoup plus touchés que d'autres et continuent de dépérir au milieu du rétablissement général. Il importe de comprendre pourquoi : expression de conditions écologiques et/ou sylvicoles particulières, ou témoin plus avancé des effets de la pollution préfigurant une évolution ultérieure encore plus défavorable ? Pour tenter de répondre à ces interrogations, un certain nombre de cas particuliers sont en cours d'étude plus approfondie, en particulier sur le plan du régime hydrique des sols et de la structure des peuplements (actuelle et plus ancienne). L'étude des effets de lisières (lisières permanentes et lisières d'origine sylvicole) est également en projet. 
Sur la base des données déjà disponibles, nous nous proposons aussi d'approfondir le déterminisme de la croissance radiale du sapin selon les conditions stationnelles et les fluctuations météorologiques, à l'aide de techniques déjà largement éprouvées par ailleurs (Fritts, 1976 ; Serre-Bachet, 1982 ; Guiot et al., 1982 ; TeSSIER, 1986 ; MONSERUD, 1986).

Reçu le 6 mai 1987.

Accepté le 12 juin 1987.

\author{
Summary \\ Present and retrospective health status of white fir (Abies alba Mill.) \\ in the Vosges (N.E. France). \\ Ecological and dendrochronological study
}

One hundred and ninety six stands were choosen within the whole natural range of white fir in the region, in order to include all the site types according to age, elevation, soil, topography, vegetation, but without prejudice to the apparent health status of the trees. Six dominant and codominant trees were selected in each stand. Their foliage density was noted and a core was taken to the pith at breast height. Thus, almost 1.200 cores, i.e. about 120,000 annual growth rings, were used for the dendrochronological study. The analysis was made difficult because of the frequent occurence of " missing rings", which must be detected in order to avoid desynchronization of the whole previous succession of rings. This was done by crossdating, using characteristic years (as far as the ring thickness is concerned) as reference years : 1870, 1893, 1916, $1922,1934$. 1948, 1956. 1961, 1976...

We tried to test the hypothesis that an increasing level of atmospheric pollution, from a date to be determined, would be the cause of a decrease in growth, which might have been developping into a real dicback for some years. From the many data available, all the rings produced at a current reference age were considered. Because of the wide diversity of the present age of the stands, the rings correspond to various calendar years. Whatever the reference age $(60$ to 110 years), no significant general trend in radial growth variation could be noticed since the beginning of the 20th century. As soon as this stagc of interpretation, it was possible to assert that the fir forest in the Vosges was not in danger of dying out before long. This was contrary to the strong fear which prevailed before this study.

However, this did not preclude that this average reality could hide more alarming special situations susceptible to spread more widely in a near future. In order to assess this possibility, the initial sample of plots was subdivided into several subsamples according to some ecological factors : elevation, soil fertility, exposure, topography. All the radial growth data were previously converted into growth indices avoiding the influence of the current age. This transformation was done in reference to the average curve « ring width/age " of the whole sample. A surprising but irrefutable conclusion emerges: none of the parameters which were used makes possible to distinguish tree populations with a behaviour significantly different from the mean. However, all the curves show that the fir forests in the Vosges have actually suffered a period of severe stress which began as early as 1973, culminated in 1976. but was almost finished in 1983 (at least, as far as radial growth is concerned). The same curves also show previous crises, comparable to the last one for their extension and severity: 1943-1951, centered on 1948, and 1917-1925, centered on 1922. As it is the case with 1976, these periods correspond to years or succession of years with a pronounced rainfall deficit.

Furthermore, the aspect of crowns and the estimated loss of needles are poor indicators of the actual vigor of the trees : the growth curves significantly deviate from the average only for needle losses above $40 \mathrm{p}$. 100 . On the contrary, sapwood width is a much more consistent parameter: it is closely correlated with the present growth, and the radial growth curves of trees in classes with different sapwood width have been deviating for 50 to 60 years, practically since the 1917-1925 crisis. 
The impact of an increasing large-scale air pollution (possible but not proved) cannot be left out, but the cause of the present crisis proves to be mainly climatic, as for the previous ones. Individual differences in tree response seem to be mostly due to the structure of the stands.

Key words: Dieback, dendrochronology, climate, Abies alba, Vosges.

\section{Références bibliographiques}

ABETZ P., 1985. Ein Vorschlag zur Durchführung von Wachstumsanalysen im Rahmen der Ursachenerforschung von Waldschäden in Südwestdeutschland. Allg. Forst.-u.J.-Ztg., 156 (9/ 10), $177-187$.

Alcubilla M., Heibl R., Rehfuess K.E., 1985. Standorstskundliche Studien zum Tannensterben : organische Inhaltsstoffe in den Nadeln befallener und gesunder Probebäume. Forstw. Cbl., $104(5), 300-312$.

Aldinger E., Kremer W.L., 1985. Zuwachsuntersuchungen an gesunden und geschädigten Fichten und Tannen auf alten Praxiskalkungsflächen. Forstw. Cbl., 104 (6), 360-372.

Athari S, 1983. Zuwachsvergleich von Fichten mit unterschiedlich starken Schadsymptomen. Allgemeine Forstzeitschrift, 26/27, 653-655.

Bauch J., Rademacher P., Berneike W., Knoth J., Michaelis W., 1985. Breite und Elementgehalt der Jahrringe in Fichten aus Waldschadensgebieten. In Forest dieback : influencing factors and their evaluation. Goslar, RFA, juin 1985. VDI-Berichte, 560, 943-959.

Becker M., 1979. Indices de climat lumineux combinant pente et cxposition. Bull. Ecol., 10 (2). 125-137.

Becker M., Lévy G., 1983. Le dépérissement du chêne. les causes écologiques (exemple de la forêt de Tronçais) et premières conclusions. Rev. Forest. fr., 35 (5), 341-356.

Becker M., 1985. Le dépérissement du sapin dans les Vosges. Quelques facteurs liés à la détérioration des cimes. Rev. forest, fr., 37 (4), 281-287.

Becker M., 1987. La santé de la forêt : le sapin témoigne. La Recherche, 191, 1096-1098.

Berchtold R., Alcubilla M., Foerst K., Rehfuess K.E., 1981. Standortskundliche Studien zum Tannensterben: Kronen - und Stammerkmale von Probebäumen aus fünf bayerischen Beständen. European Journal of Forest Pathology, 11 (4), 233-243.

CaChan P., 1974. Etude bioclimatique du massif vosgien. Bull. ENSAIA, Nancy, $16(1 / 2), 3-47+$ 1 carte.

Clauser F., 1981. Ancora sulla moria dell'abete bianco. Monti e Boschi, 32 (6), 95-97.

Cramer H.H., 1984. On the predisposition to disorders of Middle European forests. Pflanzenschutz-Nachrichten Bayer, 37 (2), 97-207.

Cramer H.H., Cramer-Middendorf M., 1984. Studies on the relationships between periode of damage and factors of climate in the Middle European forests since 1851. PflanzenschutzNachrichten Bayer, 37 (2), 208-334.

Dessureault M., 1985. Le dépérissement des arbres : nature, causes et mécanismes. Phytoprotection, 66, 71-81.

Duhamel D., 1984. Statut écologique du chêne pédonculé et du chêne sessible dans le quart nordouest de la France. Performances relatives et gestion sylvicole. Mémoire $3^{\mathrm{c}}$ année ENITEF, INRA, 58 p. + ann.

Eichkorn Th., 1986. Waschstumanalysen an Fichten in Südwestdeutschland. Allg. Forst-u.J.-Ztg., 157 (7), 125-139.

Fourchy P., 1951. Sécheresse, variations climatiques et végétation. Rev. forest. fr., 3 (1), 47-55.

FritTs H.C., 1976. Tree-ring and climate. Academic Press, 567 p.

Gerecke K.L., 1986. Zuwachsuntersuchungen an vorherrschenden Tannen aus Baden-Württemberg. Allg. Forst-u.J.-Ztg., 157 (3/4), 59-68. 
Granier A., 1981. Etudes des relations entre la section du bois d'aubier et la masse foliaire chez le Douglas (Pseudotsuga menziesii (Mirb.) Franco). Ann. Sci. for., 38 (4), 503-512.

Guiot J., Tessier L., Serre-Bachet F.. 1982. Application de la modélisation ARMA en dendroclimatologic. C.R. Acad. Sc. Paris. 294 (III), 133-135.

Hermfine M., 1984. Le dépérissement des forêts et les pluies acides. Mémoire $2^{\prime}$ année ENGREF, 84 p. + ann.

Hinckify T.M., Lasson: J.P. 1981. Radial growth in conifers and deciduous trees. A comparison. Mitteilungen der forstlichen Bundesversuchsanstalt Wien. 142. 17-56.

Hinrichisen D.. 1986. Multiple pollutants and forest dedine. Ambio, 15 (5), 258-265.

JONSSON B., SUNDBERG R., 1972. Has the acidification by atmospheric pollution caused a growth reduction in Swedish forests. A comparison of growth between regions with different soil properties. Rapporter och Uppsatser, Institutionen for Skogsproduktion, Skogshögskolan, n" 20, $46 \mathrm{p}$.

KeENan R.J., Candy S., 1983. Growth of young Eucalyptus delegatensis in relation to variation in site factors. Australian Forest Research, 13 (3/4). 197-205.

KENK G.. 1983. Zuwachsuntersuchungen in geschädigten Tannen-Beständen in Baden-Württemberg. Allgemeine Forstzeitschrift. 26/27, 650-652.

Kenk G.. Kremfr W.. Bonaventura D.. Gallus M.. 1984. Jahrring-und zuwachsanalytische Untersuchungen in erkrankten Tannenbeständen des Landes Baden-Württemberg. Mitteilungen. Forstliche Versuchs-und Forschungsanstalt, 112, 41 p.

Kienast F.. Fi.ühler H.. Schwelngruber F.H., 1981. Jahrringanalysen an Föhren (Pinus silvestris L.) aus immissionsgefährdeten Waldbeständen des Mittelwallis (Saxon. Schwciz). Mitteilungen Eidgenossische Anstalt für das forstliche Versuchswesen, 57 (4), 415-432.

KIENNE:N L.. Schuck H.J.. 1983. Untersuchungen über die Zuwachsentwicklung bei erkrankten Tannen. European Journal of Forest Pathology. 13 (5/6), 289-295.

Kramer H.. 1986. Dégâts dans les couronnes et accroissements dans les peuplements forestiers résincux frappés par le récent « dépćrissement des forêts ». Rev. forest. fr., 38 (2), 124-133.

KUISCHA N.P.. SACuS I.B.. 1962. Color tests for differentiating heartwood and sapwood in certain softwood tree species. U.S. Forest Products Laboratory, Madison, Wis., F.P.L. n" 2246, 13 p.

Landmann G.. Bonneau M.. Adrian M.. 1987. Le dépérissement du sapin pectiné et de l'épicéa commun dans le massif forestier vosgien est-il en relation avec l'état nutritionncl des peuplements? Rev forest. fr., 39 (1), 5-11.

LARSEN J.B.. 1986. Das Tannensterben: cine neue Hypothese zur Klärung des Hintergrundes dieser rätselhaften Komplexkrankheit der Weisstanne (Abies alba Mill.). Forstw. Cbl., 105 (5). $381-396$

Lévy G., Becker M.. 1987. Le dépérissement du sapin : rôle primordial de déficits d’alimentation en eau. Ann. Sci. for. 44 (4).

Mclaughlin S.B., Blasing T.J.. Mann L.K., Duvick D.N., 1983. Effects of acid rain and gaseous pollutants on forest productivity: a regional scale approach. Journal of the Air Pollution Control Association, 33 (11), 1042-1049.

McLaughuin SB., 1985. Effects of air pollution on forests. A critical review. Journal of the Air Pollution Control Association, 35 (5). 512-534.

Mitscierlich G., 1983. Untersuchungen üher dic Reakrion kranker Altannen auf die Witterung. Allgemeine Forst-und Jagdzeitung. 154 (6/7), 101-105.

Monserud R.A., 1986. Time-series analyses of tree-ring chronologies. Forest. Sci., 32 (2), 349 372.

Moosmayer H.U., 1984. Erkenntnisse über die Walderkrankung. Dargestellt an Projekten der forstlichen Versuchs-und Forschungsanstalt Baden-Württemberg. Forstw. Cbl., 103. 1-16.

Morrison I.K.. 1984. Acid rain. A review of literature on acid deposition effects in forest ecosystems. Forestry Abstracts, 45 (8), 483-506.

Pollanschütz. J., 1985. Zur Frage Nadelverluste und Kronenverlichtung bei Ficthte. Allgemeine Forstzeitung, 96 (5), 110-114.

RoL R. 1951. Le dépérissement des chênes. Rev. forest. fr., 3 (11), 707-709.

Schürt P.. Cowling E.B., 1985. Waldsterben. a general decline of forests in Central Europe : symptoms, development, and possible causes. Plant Disease, 69 (7), 548-558. 
SCitweingruber F.H.. Kontic R., Winkler-Seifert A., 1983. Eine jahrringanalytische Studie zum Nadelbaumsterben in der Schweiz. Institut fédéral de recherches forestières, rapport $\mathbf{n}^{\circ} 253$, $29 \mathrm{p}$.

Schweingruber F.H., Kontic R., Niederer M., Nippel C.A., Winkler-Seifert A., 1985. Diagnosis and distribution of conifer decay in the Swiss Rhone valley. A dendrochronological study. Berichte, Eidgenossische Anstalt für das forstliche Versuchswesen, 270, 189-192.

SChWEINGRUBER F.H., 1985. Abrupt changes in growth reflected in tree ring sequences as an expression of biotic and abiotic influences. Inventoring and Monitoring Endangered Forests, IUFRO Conference Zurich, 291-295.

Serre-BAChet F., 1982. Analyse dendroclimatologique comparée de quatre espèces de pins et du chêne pubescent dans la région de la Gardiole près Rians (Var. France). Ecologia Mediterranea, 8 (3), 167-183.

SPIEcker H.. 1986. Das Wachstum der Tannen und Fichten auf Plenterwald-Versuchsflächen des Schwarzwaldes in der Zeit von 1950 bis 1984. Allg. Forst. u.J.-Ztg., 157 (8), 152-164.

Tessier L., 1986. Approche dendroclimatologique de l'écologie de Pinus silvestris L. et de Quercus pubescens Willd dans le Sud-Est de la France. Acta (Ecologica, Eocol. Plant., 7 (21), 4, 339355.

THRFN M., 1985. Erste Ergebnisse von Wachstumanalysen an vorherrschenden und herrschenden Kiefern in Südwestdeutschland. Allg. Forst.-u.J.-Ztg., 156 (11), 215-224.

Turc L.. 1927. Note sur le dépérissement du chêne pédonculé dans les forêts du plateau nivernais. Rev. Eaux et Forêts, 65 (11), 561-565.

VINS B., 1966. Störungen in der Jahrringbildung als Fehlerquellen bei der Zuwachsbohrung. Mitt. Schweiz. Forstl. Vers.-Wes. Anst, 42 (4).

Waringi R.H., Thites W.G., Muscato D., 1980. Stem growth per unit of leaf area : a measure of tree vigor. For. Sci, 26, 112-117. 